\title{
Automated Mapping of Multiple Elements for Dose-Efficient Elemental Quantification using EFTEM
}

\author{
A. Aitouchen and P.J. Thomas \\ Gatan Inc., 5794 W. Las Positas Blvd., Pleasanton, CA 94588, USA
}

Simple two or three window techniques for EFTEM mapping provide a simple yet effective method for the rapid generation of elemental distribution maps. While this technique is not as powerful as the EFTEM spectrum imaging approach, it provides a convenient approach for acquiring elemental maps with high pixel densities and is particularly suitable for samples which have few unknowns where a qualitative elemental distribution is required. As such, it is the method of choice for rapid generation of EELS maps using an imaging filter, particularly for operators in environments where generating element-specific distribution maps with fast turnaround is of high priority.

The multi-window technique does however provide some challenges for the inexperienced operator. Generally, the operator must manually determine the optimal acquisition parameters for each individual edge of interest, (e.g., camera exposure, CCD binning and energy window positions must be simultaneously optimized). Further, since these parameters must be determined on an edge-by-edge basis, the overall beam dose on the specimen is often much higher than required. Finally, the operator typically wants to acquire multiple maps, including the necessary reference low-loss images, in a single automated acquisition thus ensuring that the data is acquired in close spatial registry and with minimal overall dose using fixed illumination conditions.

This work has concentrated on developing the necessary tools to allow multi-map acquisition to be performed in a fast, fully-automated, reproducible and potentially quantitative manner, by dynamically changing the acquisition parameters as the acquisition proceeds [1]. Key to achieving this goal are a number of developments, including:

1. A highly efficient auto-exposure and auto-binning algorithm for dynamically determining the optimal exposure for each core-loss edge with a minimal dose.

2. Software to facilitate the acquisition of multiple maps in sequence, and for the storing and recalling of predetermined element map lists, facilitating ease of use and reproducibility.

3. A visual interface to facilitate energy window placement and to avoid edge overlap.

The combination of the above features allows the acquisition of elemental maps with near optimal set-up parameters for a number of elements and/or ESI images over a wide energy-loss range in a fast, automated manner, requiring little more from the user than setting up the illumination conditions and specifying the elements of interest. An example of a data-set acquired in this way is shown in Figure 1 for a semiconductor device sample. The total acquisition time was under 3.5 minutes, including all test exposures and settling delays, which is considerably faster than if it was performed by even an experienced user. Since the data is acquired under identical illumination conditions, along with the corresponding zero-loss 
information, the three window maps can be quantified using standard quantification techniques using the corresponding ionization cross-sections [2].

\section{References}

[1] P. J. Thomas and P.A. Midgley, Ultramicroscopy 88 (2001) 187-194

[2] Hofer F., et al, Ultramicroscopy 67 (1997) 83-103
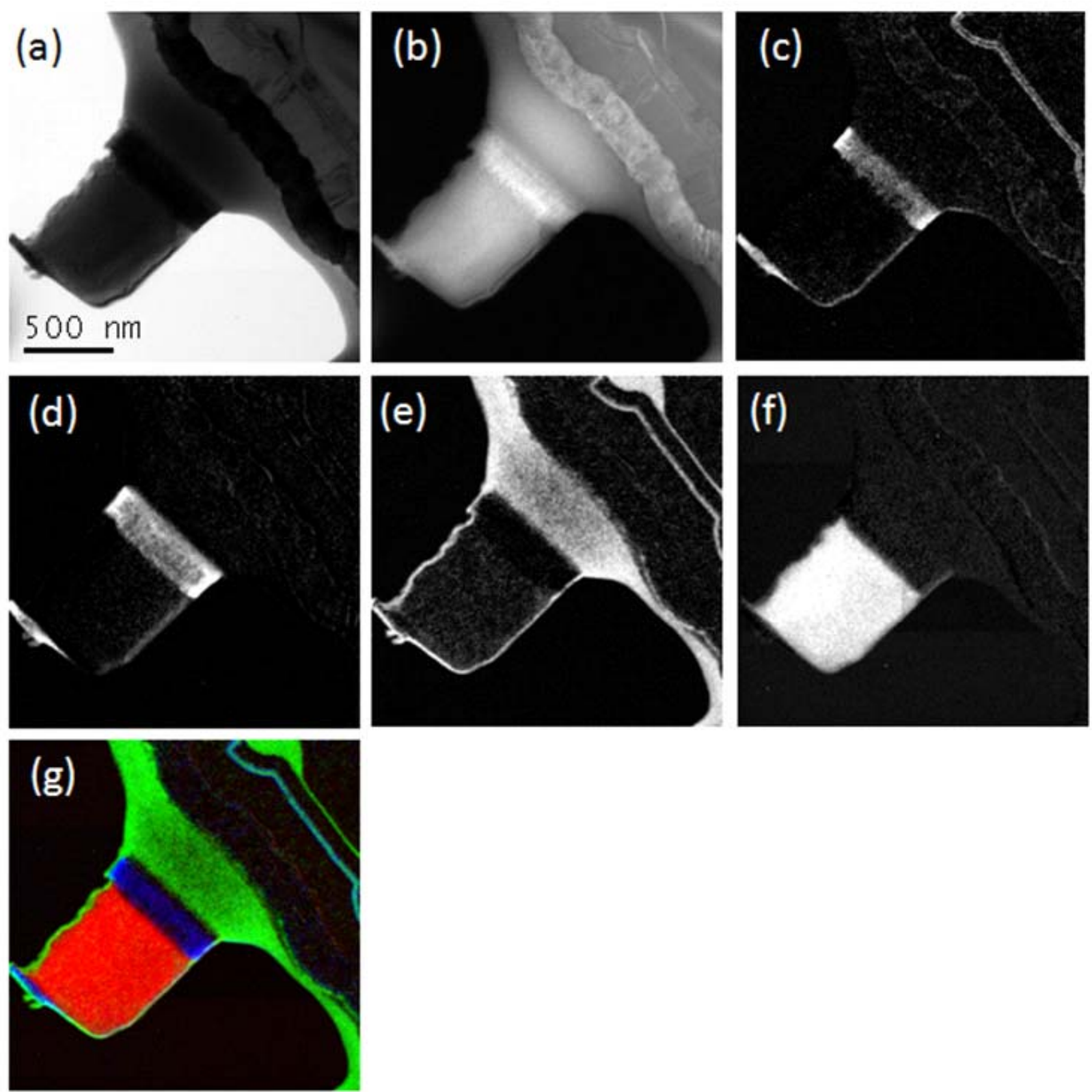

Fig.1. Automated EFTEM multi-element mapping, acquired using dynamic exposure and binning determination to optimize CCD exposure conditions. The zero-loss, relative thickness, $\mathrm{N} \mathrm{K}(401 \mathrm{eV})$, Ti L $(455 \mathrm{eV}), \mathrm{O} \mathrm{K}(532 \mathrm{eV})$, and Al K $(1550 \mathrm{eV})$ maps were all acquired under identical illumination conditions and as a single multi-map acquisition (a-f respectively). An RGB composite of $\mathrm{Al}, \mathrm{O}$, and $\mathrm{N}$ respectively is shown in $\mathrm{g}$ ). Total acquisition time was $<200$ s (including all pre and post edge images). 